\title{
Does dual-energy abdominal computed tomography increase the radiation dose to patients: a prospective observational study
}

\author{
Dilip John ${ }^{B, C, E, F}$, Athira R. ${ }^{B}$, Swathigha Selvaraj ${ }^{C, F}$, Rupa Renganathan ${ }^{A, E}$, Kannan Gunasekarann, \\ Venkatesh Kasi Arunachalam ${ }^{\mathrm{A}, \mathrm{D}, \mathrm{E}}$
}

Department of Radiology, Kovai Medical Center and Hospital, Coimbatore, India

\begin{abstract}
Purpose: The aim of our study was to compare single-energy (SECT) and dual-energy (DECT) abdominal computed tomography (CT) examinations in matched patient cohorts regarding the differences in effective radiation dose (ERD) and image quality performed in a third-generation dual-source computed tomography (DSCT) scanner.

Material and methods: Our study included 100 patients, who were divided randomly into 2 groups. The patients included in Group A were scanned by SECT, and Group B members were scanned by DECT. Volume CT dose index $\left(\mathrm{CTDI}_{\mathrm{vol}}\right)$, dose length product (DLP), and ERD for venous phase acquisition were recorded in each patient and were normalised for $40 \mathrm{~cm}$. Analyses were performed by using statistical software (SPSS version 20.0 for windows), and Bonferroni correction for multiple comparisons was applied for $p$-values and confidence intervals.

Results: Average ERD based on DLP values normalised for $40 \mathrm{~cm}$ acquisition were obtained for both Group A and Group B. The mean ERD for Group A was $11.89 \mathrm{mSv}$, and for group B it was $6.87 \mathrm{mSv}$. There was a significant difference in these values between Group A and Group B as shown by a $p$-value of $<0.001$. On subjective and objective analysis, there was no statistically significant difference in image quality between the 2 groups.

Conclusions: The protocols in third-generation DSCT using dual-energy mode resulted in significant reductions in the effective radiation dose (by approximately 58\%) compared to SECT in routine abdominal examination in matched cohorts. Therefore, the quantitative imaging potential of DECT can be utilised in needed patients with decreased radiation dose in third-generation DSCT.
\end{abstract}

Key words: radiation dose, third generation, DSCT, DECT, SECT, image quality.

\section{Introduction}

Dual-energy computed tomography (DECT) has been increasingly used in abdominal studies because it helps in material decomposition and also facilitates hepatic iron load differentiation, gallstones, and renal calculi. Abdominal spectral DECT has been used to analyse iodine uptake for characterisation of hepatic, renal, and adrenal lesions, assessment of bone mineral density of the lumbar spine, and to decrease beam-hardening artefacts. The postprocessing of DECT data sets has also shown potential to improve image contrast and increase iodine signal intra- vascularly in suboptimal contrast conditions. Such techniques can also be used to reduce the volume of contrast in contrast-enhanced CT.

In the current era, because of its increased applications, DECT is routinely performed. However, the common misconception among clinicians is that DECT increases the radiation dose because the patient is scanned by dual energy levels [1]. We need convincing evidence to suggest there is no radiation dose penalty associated with DECT, so that all DECT advanced applications can be used in routine clinical practice. Previous studies have shown that second-generation DECT can be performed

Correspondence address:

Venkatesh Kasi Arunachalam, Department of Radiology, Kovai Medical Center and Hospital, Coimbatore, India, e-mail: drkasivenkatesh@yahoo.co.in

Authors' contribution:

A Study design · B Data collection · C Statistical analysis · D Data interpretation - E Manuscript preparation · F Literature search · G Funds collection 
with no or only minimal dose penalty compared to singleenergy CT (SECT) acquisition [2,3]. With the recent third-generation DSCT system, which has substantially higher X-ray tube current reserves, additional dualenergy tube voltage combinations, and a thicker tin filter for both tubes, an increase in spectral separation and further reduction in the effective radiation dose to the patient is possible.

The purpose of this study was to calculate and compare the effective radiation dose of SECT and DECT of abdomen examinations performed with third-generation dual-source CT (DSCT).

\section{Material and methods}

The study was approved by the local ethics committee and written informed consent was obtained from each participant. This was an observational study and was done during the period October 2019 to March 2020. Patients with age $>18$ years, who underwent contrast-enhanced CT abdomen examinations were included in the study. Patients with absolute contraindications for iodinated contrast and scans with movement artefacts were excluded from the study.

A total of 100 patients were included and divided into 2 groups of 50 each (Group A and Group B). Groups were matched by gender ( 25 males and 25 females in each group) and body mass index (BMI) to allow for a direct comparison of study groups. The patients included in Group A were scanned by SECT, and Group B members were scanned by DECT.

\section{Computed tomography technique (image acquisition and post processing)}

Scans of groups A and B were performed on third-generation DSCT (Somatom Force, Siemens Healthcare Sector, Forchheim, Germany). All the patients in Group A and Group B underwent scans during the venous phase from the level of the dome of the diaphragm to the lower end of the ischial tuberosity in SECT and DECT, respectively. These patients underwent plain and arterial phases whenever there was a need according to the clinical scenario and the above-mentioned phases in both groups were performed with SECT in our institution.

Dose-optimised SECT was performed using online dose modulation (CARE Dose 4D) and automatic voltage control (Care kV, Siemens). The scan was performed with a detector collimation of $192 \times 0.6 \mathrm{~mm}$ in craniocaudal direction and a pitch of 1 (Figures 1-3). The DECT scan was performed by using 2 different tubes voltages $(100 \mathrm{kV}$ and tin filtered $150 \mathrm{kV}$ [Sn $150 \mathrm{kV}$ ]) and online dose modulation (CARE Dose 4D, Siemens). The scan was acquired with a detector collimation of $128 \times 0.6 \mathrm{~mm}$ in craniocaudal direction and a pitch of 1 (Figures 4-6). The acquisition parameters are detailed in Table 1.
Iohexol $350 \mathrm{mg} / \mathrm{ml}$ was used as a contrast agent, and a dose of $1 \mathrm{ml} / \mathrm{kg}$ was injected at a flow rate of $4 \mathrm{ml} / \mathrm{s}$ followed by a $30 \mathrm{ml}$ saline flush using an automated dualsyringe power injector through an 18 gauge IV access placed in the right median cubital vein. The timing of the scan was performed using a bolus tracking software application. The region of interest (ROI) was placed in the abdominal aorta, when HU of 200 was reached, and the scan was triggered with a delay of 4 seconds for early arterial phase, 15 seconds after bolus tracking for late arterial/ early portal venous phase and 50-60 seconds delay for the venous phase.

The images in both SECT and DECT were reconstructed using a standard soft tissue reconstruction kernel (Br36) using modern iterative reconstruction technique, namely the Advanced Modeled Iterative Reconstruction (ADMIRE) algorithm, with slice thickness of $0.6 \mathrm{~mm}$. Images with $3 \mathrm{~mm}$ thickness in axial, sagittal, and coronal planes were also generated. A single image series was created for each DECT examination using standard linear blending from the spectral datasets (weighting factor of 0.5 ) which corresponds to a $120 \mathrm{kV}$ scan. After completion of image acquisition and reconstruction, images were transferred to a dedicated workstation (syngo.via, Siemens) for further analysis.

\section{Radiation dose analysis}

Patient protocols for each patient in the 2 groups were analysed, and the tube current, volume CT dose index $\left(\mathrm{CTDI}_{\mathrm{vol}}\right)$, dose length product (DLP), and acquisition length in $\mathrm{cm}$ for venous phase acquisition were recorded.

To account for variations in scan acquisition length between patients, DLP was normalised for a typical abdominal acquisition covering $40 \mathrm{~cm}$. The ERD estimate was calculated for each patient by multiplying the DLP with an abdomen-specific conversion coefficient $\kappa$ of $0.017 \mathrm{mSv} /[\mathrm{mGy} \cdot \mathrm{cm}][4,5]$. Previous studies have demonstrated that this conversion factor can be used for both SECT and DECT acquisition [6].

\section{Subjective image analysis}

Subjective image analysis was done by a radiologist with more than 10 years' experience in abdominal cross-sectional imaging using the 5-point scale for anatomical details and artefacts (Table 2) in a $3 \mathrm{~mm}$ axial slice image dataset. This scoring criteria was developed on the basis of previous studies done by Li et al. [7] and De Ceccoa et al. [8].

\section{Objective image quality evaluation}

The image quality was objectively evaluated using the contrast-to-noise ratio (CNR) and figure-of-merit (FOM) values. Initially $\mathrm{CT}$ attenuation values were measured by placing a circular ROI over the liver (ROI size, 50-100 $\mathrm{mm}^{2}$ ), spleen (ROI size, $50-100 \mathrm{~mm}^{2}$ ), portal vein (ROI size, 


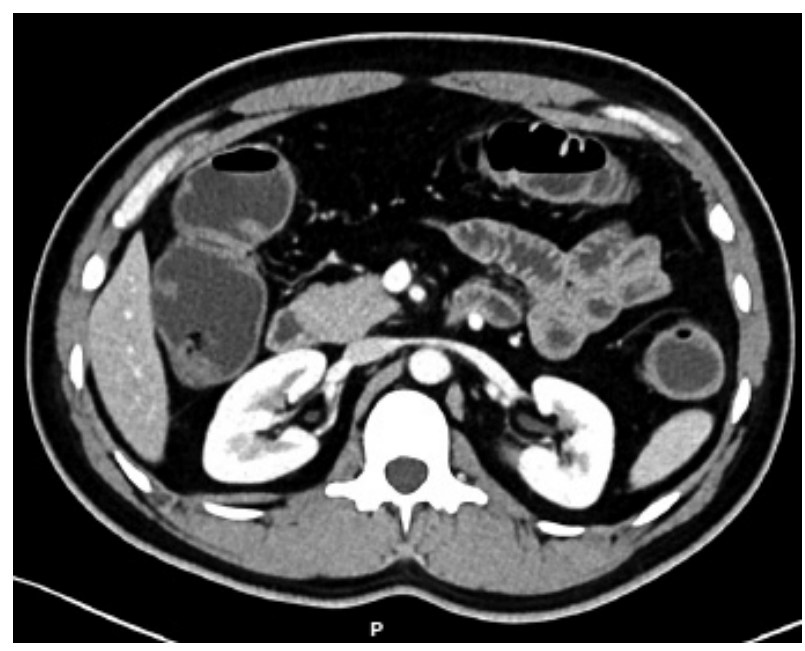

Figure 1. Axial abdominal contrast enhanced venous phase computed tomography image, acquired in single-energy mode, of a 34-year-old female with a body mass index of $22.15 \mathrm{~kg} / \mathrm{m}^{2}$

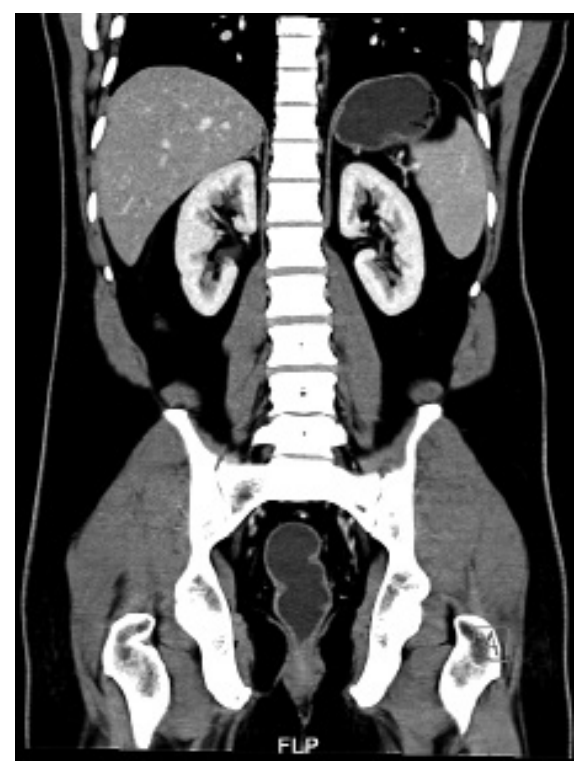

Figure 2. Coronal and sagittal reformatted abdominal contrast enhanced venous phase computed tomography images acquired in single-energy mode of the same patient
30-60 $\mathrm{mm}^{2}$ ), erector spinae muscle (ROI size, 50-100 $\mathrm{mm}^{2}$ ), and anterior abdominal wall subcutaneous fat (ROI size, $30-100 \mathrm{~mm}^{2}$ ) on $3 \mathrm{~mm}$ axial images of all 100 data sets. Areas with focal heterogeneity of contrast enhancement, focal calcification, and adjacent anatomical structures were excluded to facilitate homogenous measurements. The measurements were repeated 3 times to avoid data inaccuracies, and the mean values were calculated. The image noise was defined as the standard deviation of the fat.

The contrast-to-noise ratios (CNR) of the liver, spleen, and portal vein were measured by dividing the absolute difference of the attenuations within the ROI and the

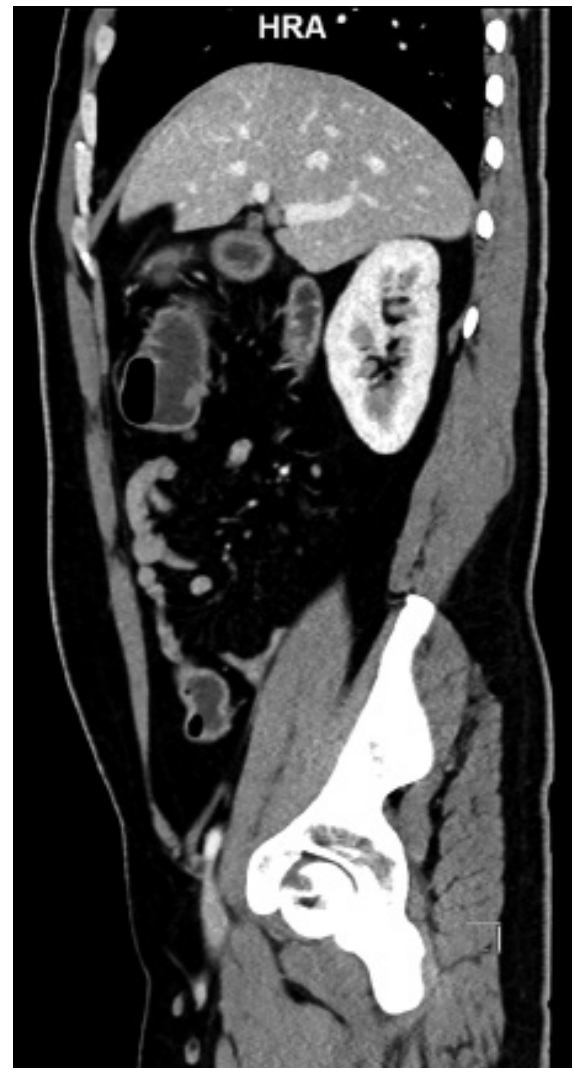

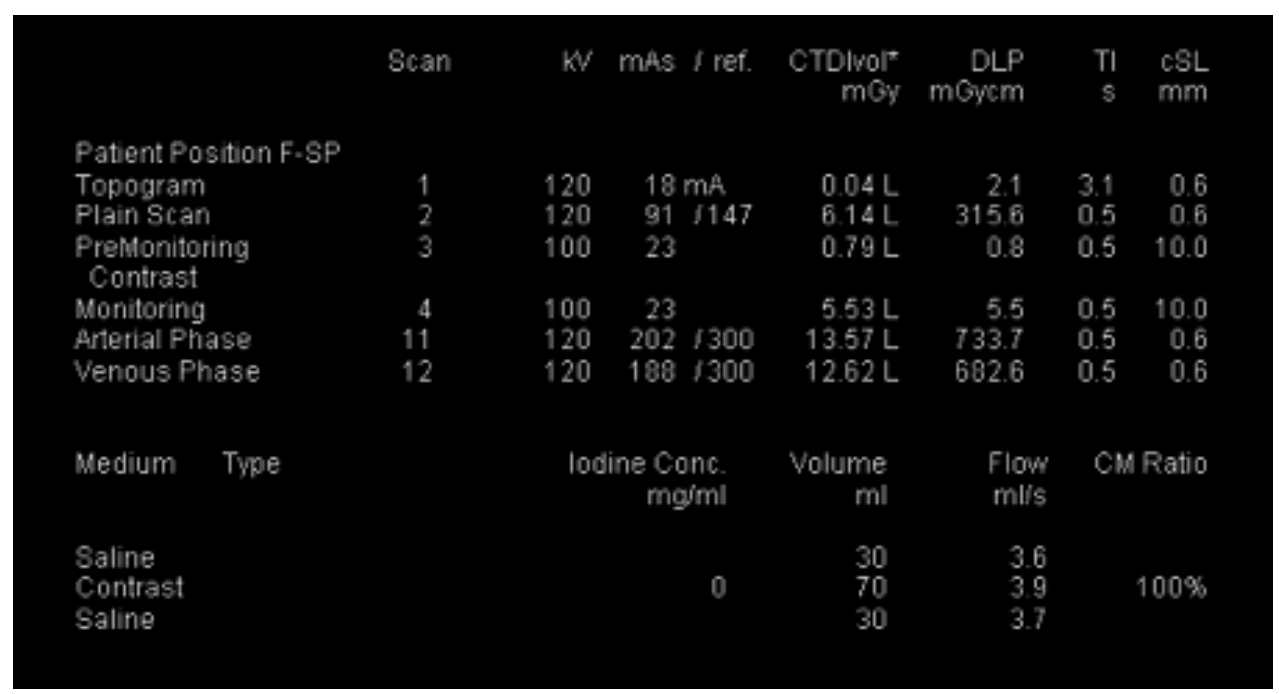

Figure 3. Dose chart of the above-mentioned patient done in single-energy computed tomography 


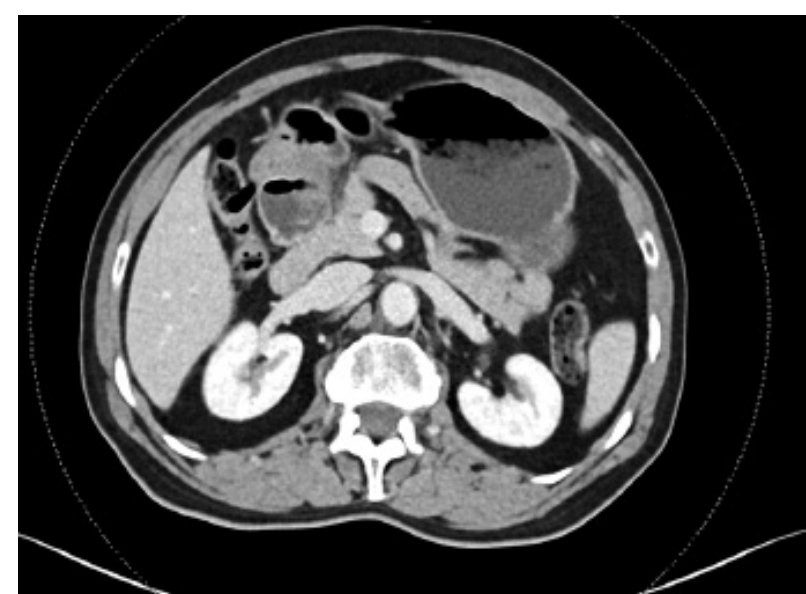

Figure 4. Axial abdominal contrast-enhanced venous phase computed tomography images acquired in dual-energy mode of a 60 -year-old female with a body mass index of $22.3 \mathrm{~kg} / \mathrm{m} 2$
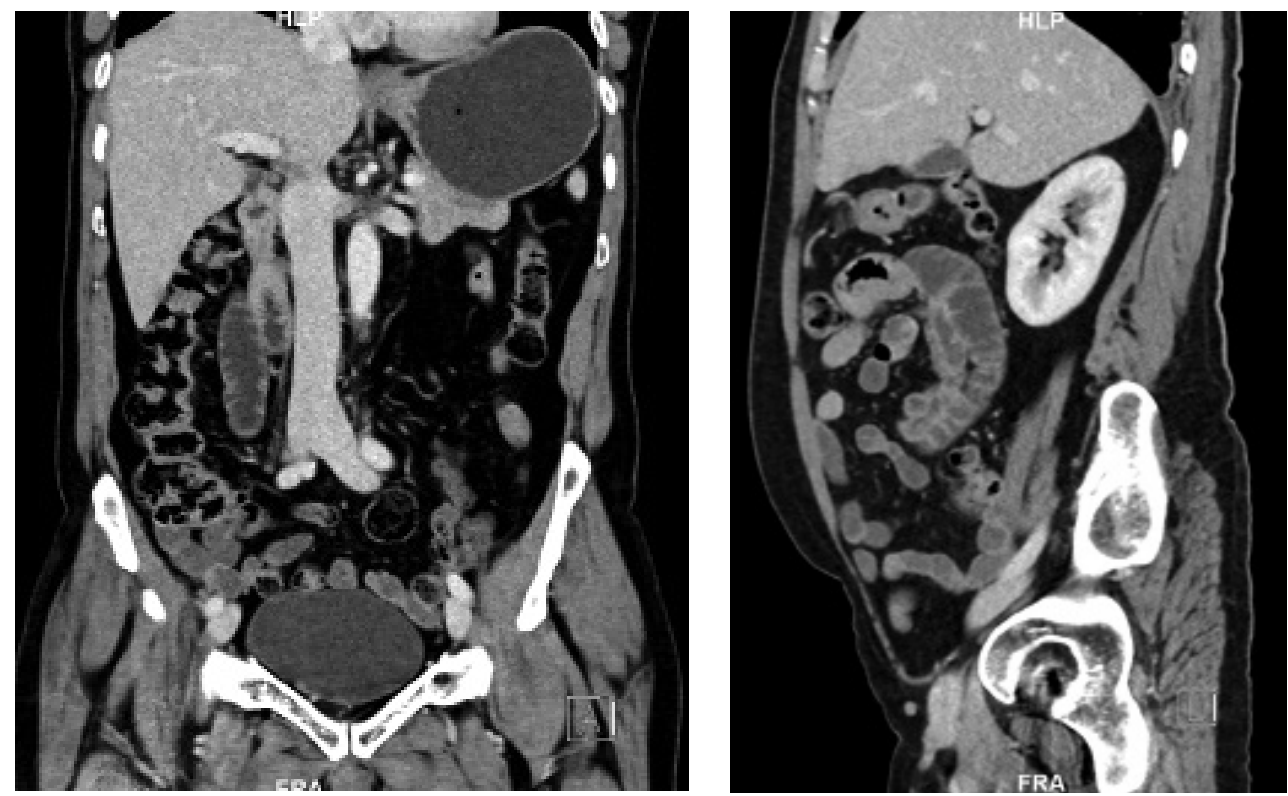

Figure 5. Coronal and sagittal reformatted abdominal contrast enhanced venous phase computed tomography images of the same patient

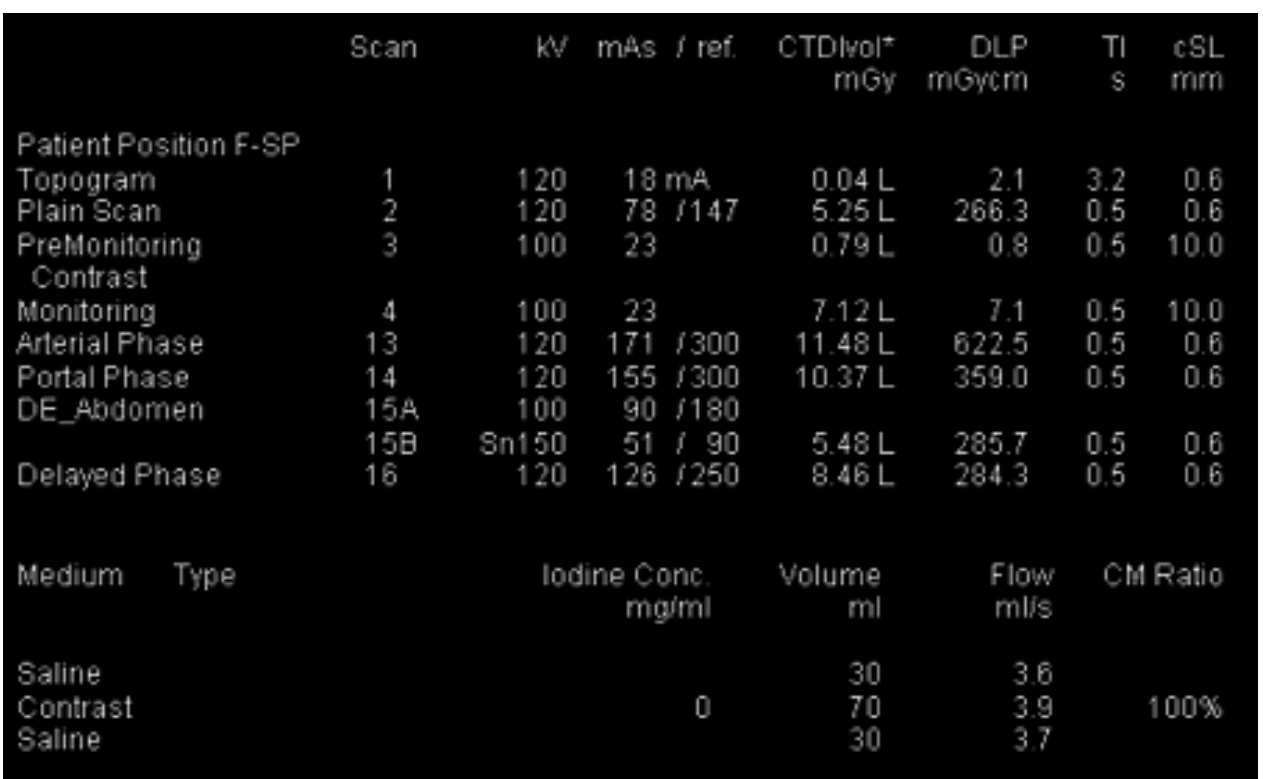

Figure 6. Dose chart of the above-mentioned patient done in dual-energy computed tomography 
Table 1. Acquisition parameters of SECT (Group A) and DECT (Group B)

\begin{tabular}{|c|c|c|}
\hline Parameter & Group A & Group B \\
\hline Acquisition mode & SECT & DECT \\
\hline Tube voltage (kV) & 120 & $90 / \mathrm{Sn} 150$ \\
\hline Tube current (ref. mAs) & 300 & $180 / 90$ \\
\hline Pitch & 1 & 1 \\
\hline Rotation time (s) & 0.5 & 0.5 \\
\hline Collimation & $192 \times 0.6$ & $128 \times 0.6$ \\
\hline Section thickness (mm) & $0.6 \mathrm{~mm}$ axial, $3 \mathrm{~mm}$ in all three planes & $0.6 \mathrm{~mm}$ axial, $3 \mathrm{~mm}$ in all three planes \\
\hline Iterative reconstruction algorithm & ADMIRE, strength 3 & ADMIRE, strength 3 \\
\hline Increment (mm) & 1 & 1 \\
\hline Kernel & $\mathrm{Br} 36$ & $\mathrm{Br} 36$ \\
\hline Linear blending in dual-energy mode & - & $50 \% / 50 \%(90 / 150 \mathrm{Kv})$ \\
\hline Tin filter & - & Selective photon shield II \\
\hline
\end{tabular}

ADMIRE - advanced modelled iterative reconstruction

Table 2. Subjective image analysis criteria based on artefacts and anatomical details

\begin{tabular}{|c|l|l|} 
Grade & Artifacts & Anatomical details and lesions \\
\hline 1 & Severe artefacts & Organs - not assessable, lesions - not visible \\
\hline 2 & Artefacts affecting diagnostic information & Anatomical details of organs - not clearly visible, lesions - not clear \\
\hline 3 & Obvious artefacts but acceptable & Anatomical details of organs and lesions - seen to an acceptable extent \\
\hline 4 & Artefacts were seen in other organs not interfering with the diagnosis & Organs and lesions - seen clearly \\
\hline 5 & Minimum or no artefacts & Organs and lesions - seen clearly \\
\hline
\end{tabular}

with repeated measures analysis of variance if data were normally distributed according to the Shapiro-Wilk test. The Kruskal-Wallis 2-way analysis of variance was used if data were not normally distributed. If the effective dose was not normally distributed, the mean difference between ERD in Group A and Group B was tested through non-parametric Mann-Whitney $U$ test.

\section{Results}

The mean age of sample Group A was 47.92 years and of Group B was 54.72 years. The age ranged between 19 and 78 years. The majority of the patients were between the age of 41 and 60 years. The mean BMI in Group A was $22.47 \mathrm{~kg} / \mathrm{m}^{2}$ and in Group B it was $22.47 \mathrm{~kg} / \mathrm{m}^{2}$ with a range of between 15.6 and $29.3 \mathrm{~kg} / \mathrm{m}^{2}$. Because the patient cohorts were matched by gender and BMI, no significant differences regarding these parameters $(p>0.99)$ were observed, although the standard deviations varied (Table 3 ).

Average effective radiation dose based on DLP values normalised for $40 \mathrm{~cm}$ acquisition was obtained for both Group A and Group B (Table 4).

The mean CTDI volume for Group A was 13.41 and for Group B it was 7.94. The mean DLP for Group A was 704.53 and for Group B it was 404.55. The mean ERD for Group A was $11.89 \mathrm{mSv}$ and for group B it was $6.87 \mathrm{mSv}$. There was a significant difference in these val- ues between Group A and Group B as shown by a $p$-value of $<0.001$. The average effective radiation dose based for DLP values normalised for $40 \mathrm{~cm}$ acquisition was lowest in group B, with a significant difference compared to group $\mathrm{A}(p<0.001)$.

\section{Subjective score}

The subjective score was analysed using a 5-point scale ranging from grade 1 to 5 , where 1 was the worst and 5 was the best possible score. The mean subjective score obtained for the 2 groups ( $\mathrm{A}$ and $\mathrm{B}$ ) were $4.38 \pm 0.52$ and $4.4 \pm 0.53$ (mean $\pm \mathrm{SD}$ ), respectively. The $p$-value for individual differences of subjective score between Group A and Group B is 0.32 , indicating that the difference in image quality was not statistically significant between the 2 groups (Table 5 ).

\section{Objective image quality evaluation}

There was no statistically significant difference in image noise between the 2 groups. The highest CNR and FOM values for liver, spleen, and portal vein were noted in the dual-energy group. However, the differences in CNR and FOM values of liver, spleen, and portal vein between both groups were not statistically significant. A detailed list of all objective image quality parameters is shown in Table 6 . 
Table 3. Statistical analysis for age, height, weight, and BMI for Groups A and B

\begin{tabular}{|c|c|c|c|c|c|}
\hline Parameter & Number of samples & Mean & Standard deviation & Standard error mean & $p$-value (A vs. B) \\
\hline \multicolumn{6}{|l|}{ Age } \\
\hline Group A & 50 & 47.92 & 16.070 & 2.273 & \multirow[t]{2}{*}{0.019} \\
\hline Group B & 50 & 54.72 & 12.022 & 1.700 & \\
\hline \multicolumn{6}{|l|}{ Height (cm) } \\
\hline Group A & 50 & 160.10 & 10.363 & 1.466 & \multirow[t]{2}{*}{0.027} \\
\hline Group B & 50 & 164.16 & 7.506 & 1.062 & \\
\hline \multicolumn{6}{|l|}{ Weight (kg) } \\
\hline Group A & 50 & 57.770 & 11.4706 & 1.6222 & \multirow[t]{2}{*}{0.2} \\
\hline Group B & 50 & 60.540 & 9.9662 & 1.4094 & \\
\hline \multicolumn{6}{|l|}{$\mathrm{BMI}\left(\mathrm{kg} / \mathrm{m}^{2}\right)$} \\
\hline Group A & 50 & 22.4760 & 3.27128 & .46263 & \multirow[t]{2}{*}{0.993} \\
\hline Group B & 50 & 22.4700 & 3.27721 & .46347 & \\
\hline
\end{tabular}

Table 4. Statistical analysis for CTDI ${ }_{\text {vol’ }}$ DLP, and effective dose for Groups A and B

\begin{tabular}{|c|c|c|c|c|c|}
\hline Parameter & Number of samples & Mean & Standard deviation & Standard error mean & $p$-value (A vs. B) \\
\hline \multicolumn{6}{|l|}{$\mathrm{CTDI}_{\mathrm{vol}}$} \\
\hline Group A & 50 & 13.4152 & 4.16509 & 0.58903 & \multirow[t]{2}{*}{0.000} \\
\hline Group B & 50 & 7.9440 & 2.39462 & 0.33865 & \\
\hline \multicolumn{6}{|l|}{ DLP } \\
\hline Group A & 50 & 704.536 & 243.7485 & 34.4712 & \multirow[t]{2}{*}{0.000} \\
\hline Group B & 50 & 404.550 & 128.7641 & 18.2100 & \\
\hline \multicolumn{6}{|l|}{ Effective dose } \\
\hline Group A & 50 & 11.892506 & 4.1329150 & 0.5844824 & \multirow[t]{2}{*}{0.000} \\
\hline Group B & 50 & 6.877350 & 2.1889903 & 0.3095700 & \\
\hline
\end{tabular}

Table 5. Mean subjective score and SD in Group A and Group B

\begin{tabular}{|l|c|c|}
\hline Subjective score & Mean & SD \\
\hline Group A & 4.38 & 0.52 \\
\hline Group B & 4.4 & 0.53 \\
\hline
\end{tabular}

Paired $t$-test; $p$-value -0.322223 ; non-significant

Table 6. Objective image quality parameters

\begin{tabular}{|c|c|c|c|c|}
\hline Parameter & Group A & Group B & $p$-value & Significant (S)/Non-significant (NS) \\
\hline Image noise & $8.45 \pm 2.50$ & $8.40 \pm 2.06$ & 0.945 & NS \\
\hline \multicolumn{5}{|c|}{ Contrast-to-noise ratio (CNR) } \\
\hline Liver & $5.19 \pm 3.06(1.8-11.1)$ & $4.88 \pm 2.43(1.8-12)$ & 0.73 & NS \\
\hline Spleen & $6.33 \pm 3.49(2.5-12.3)$ & $5.78 \pm 2.63(2.6-13.2)$ & 0.553 & NS \\
\hline Portal vein & $11.72 \pm 6.17(3.2-24.2)$ & $10.43 \pm 4.62(3.1-24.4)$ & 0.462 & NS \\
\hline \multicolumn{5}{|c|}{ Figure of merit CNR } \\
\hline Liver & $4.21 \pm 6.57(0.3-15.9)$ & $5.070 \pm 7.00(0.4-30.3)$ & 0.69 & NS \\
\hline Spleen & $5.28 \pm 6.67(0.5-24.9)$ & $6.76 \pm 8.44(0.6-36.7)$ & 0.543 & NS \\
\hline Portal vein & $18.27 \pm 22.94(1.2-65.3)$ & $22.38 \pm 28.16(2.9-125.6)$ & 0.616 & NS \\
\hline
\end{tabular}

Data are given as mean \pm standard deviation and range in parenthesis. 


\section{Discussion}

With the current technological advancements, DECT has gained worldwide acceptance. In abdominal imaging, DECT examinations are mainly considered for oncological staging and follow-up, evaluation of vascular pathology, preoperative workups, and evaluation of gastrointestinal bleeding. DECT also has potential in radiation dose reduction.

The previously used first- and second-generation DSCT had certain disadvantages, such as poor spatial resolution, motion-related artefacts, and increased noise and radiation dose [10-13]. These problems were addressed in the third-generation DSCT. Although there are different software and hardware tools among the thirdgeneration DSCT scanners for radiation dose reduction, there is much apprehension regarding the same among the clinicians [1]. We performed this study to compare the radiation dose in single- and dual-energy CT of abdomen examinations in matched patient cohort.

\section{Evaluation of radiation dose}

The results of our study show that dual-energy CT can be performed without radiation dose penalty compared to SECT with a third-generation DSCT scanner. When we evaluated a single venous phase in a matched patient cohort in third-generation DSCT using dual-energy mode, a dose reduction of $57.8 \%$ was achieved compared to single-energy CT.

The most efficient way to reduce radiation dose in CT is by adapting the scan parameters to the patient's anatomy. Centring the patient correctly, using the right protocols, and adjusting the X-ray tube output to the patient's size and shape help to minimize radiation exposure. Thirdgeneration DSCT has control mechanisms that automatically adjust the radiation dose to the patient's anatomy. This technique is called CARE Dose4D, which automatically adapts the tube current to the size and shape of the patient and achieves optimal tube current modulation $[14,15]$. In this the tube current is first varied on the basis of a topogram, by comparing the actual patient to a "standard-sized" patient. The tube current as expected is increased for larger patients and reduced for smaller patients. The differences in attenuation in distinct body regions are also taken into account. Also, the real-time angular dose modulation measures the actual attenuation in the patient during the scan and adjusts the tube current accordingly - not only for different body regions, but also for different angles during rotation. The improved X-ray tube current capabilities of third-generation DSCT allow usage of a lower tube voltage (i.e. $100 \mathrm{kV}$ ) in abdominal imaging.

The next most important factor useful in reducing radiation dose in third-generation DSCT is tin filters. In third-generation DSCT, each X-ray source has a tin filter that filters out unnecessary photons for powerful low-dose scanning. It helps to achieve an optimized spectrum for dose efficiency and helps to deliver powerful low-dose scanning at the level of conventional X-ray examinations. Also, the third-generation DSCT systems are equipped with digital detectors (Stellar infinity detectors), which are more sensitive to electron influx. These detectors can measure smaller signals over a wider dynamic range, which directly enhances CT image quality especially for applications with extremely low signal levels. Such extremely low signal levels play an important role when scanning large patients and in low-dose scans, as well as in the low-kV datasets of dual-energy examinations. All the above-mentioned factors result in a significant reduction in radiation dose in third-generation dual-source CT. Our results for radiation doses with third-generation dual-energy CT $(6.87 \pm 4.37 \mathrm{mSv})$ averaged for 40 - $\mathrm{cm}$ acquisitions are well below recently reported results associated with contrast-enhanced routine abdomen-pelvis CT (median of $16 \mathrm{mSv}$ ) [14] and are in line with the suggestions $(10 \mathrm{mSv})$ from a patient education website [15].

Some of the prior studies that have investigated the radiation dose delivered by DECT compared to SECT have shown varying results. De Cecco et al. assessed secondgeneration DSCT protocols and compared SECT and DECT [4] and reported a small but significant increase in the radiation dose of the order $1 \mathrm{mSv}$ with dual-energy CT. No significant differences in radiation dose between abdominal second-generation SECT and DECT was found in the study by Primak et al. [16]. A significant decrease in radiation dose with DECT compared to SECT in patients who underwent abdominal second-generation SECT and DECT for hepatocellular carcinoma screening was observed in a study by Purysko et al. [17]. In their study they observed a $37 \%$ reduction in overall dose with DECT compared to SECT. In our study we observed that thirdgeneration DECT showed a significant decrease in radiation dose (57.8\%) compared to SECT. Thus, dual-energy CT can be implemented in routine clinical use without negatively impacting image quality while lowering the radiation dose to the patient.

In our study, we observed the lowest radiation doses with DECT in third-generation DSCT. The mean effective radiation dose in second-generation dual-source CT in the study by Purysko et al. was $12.97 \mathrm{mSv}$ [17]. However, in their study the results were not normalised for $40 \mathrm{~cm}$ acquisition. In a study by De Cecco et al., the mean radiation dose in second-generation dual-source CT normalised for $40 \mathrm{~cm}$ was $10.2 \mathrm{mSv}$ [4]. In our study the mean effective radiation dose was $6.88 \mathrm{mSv}$. Our results were similar to those in the study by Wichmann et al. [3]. They concluded that with both second and third DSCT generations, abdominal DECT can be routinely performed without radiation dose penalty compared to SECT, while third-generation DSCT shows improved dose efficiency. David et al. in their study observed that 

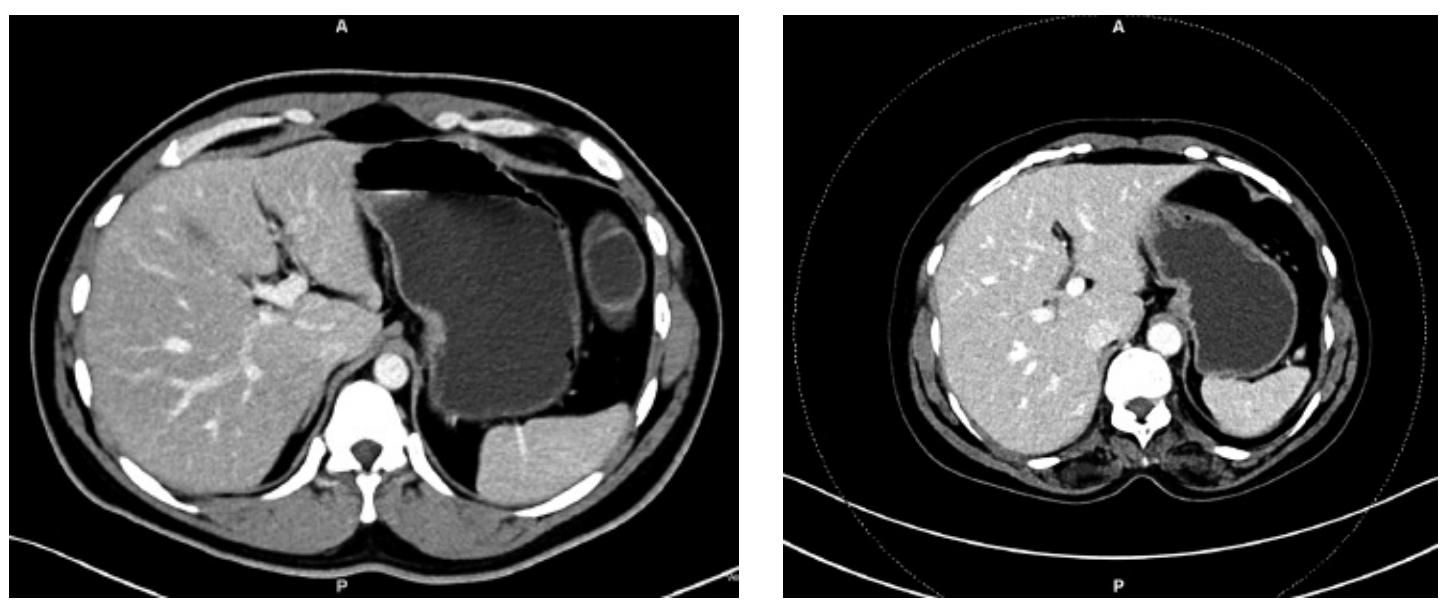

Figure 7. Axial sections of abdomen in single-energy computed tomography (A) and dual-energy computed tomography (B) with grade 5 image quality

the mean dose-length product (DLP) and effective dose of DECT were significantly lower than the DLP and effective dose of SECT $(p<0.05)$ [18]. The mean radiation dose in their study was $6.1 \mathrm{mSv}$. A reliable explanation of this reduced radiation dose could be the improved tube current capabilities in third-generation DSCT.

The other factor that plays an important role in dose reduction was modern iterative reconstruction algorithms. In our CT scan the latest generation of Siemens iterative reconstruction, called the ADMIRE algorithm, was used for the reconstruction of both SECT and DECT images. It was applied at a strength of 2 (ADMIRE 2) out of a maximum strength of 5 . This level 2 strength represents a low to medium level of noise suppression due to iterative reconstruction. ADMIRE reduces image noise on CT images and maintains the spatial resolution and contrast-to-noise ratio. Some of the studies in the previous literature have mentioned that ADMIRE plays an important role in reducing radiation dose by compensating image noise with its post processing capabilities [19,20].

Further reduction in radiation does is possible in DECT scans in multiphasic examination, by utilising virtual non-contrast images and avoiding true unenhanced phase/plain scan. In a study by De Cecco et al., it was observed that by avoiding true unenhanced acquisition the radiation dose can be reduced by $32.9 \%$ [8]. Similarly, in a study by Chen et al., a decrease in radiation dose of $57 \%$ was achieved by omitting true unenhanced images in a single-phase DECT scan [21].

\section{Evaluation of subjective criteria}

The basic aim of the protocols used in this study was to generate good quality images with minimal radiation exposure. In our study we used the 5-point scoring system for assessing the subjective criteria. These scoring criteria were developed on the basis of the previous studies done by De Ceccoa et al. [8] and Li et al. [7]. All the images assessed had a score of 3 or more, indicating that they were suitable for interpretation (Figure 7). The $p$-value for in- dividual differences of subjective score between Group A and Group B was 0.32, indicating that the difference in image quality was not statistically significant between the 2 groups. This result is similar to the study done by Wichmann et al. [3], which showed no significant difference in image quality between single- and dual-energy scans. In a study by Tawfik et al., no significant difference in subjective image quality was observed between SECT and DECT protocols [22].

\section{Evaluation of objective image quality}

Objective image quality assessment revealed the highest values for CNR and FOM for all the assessed organs in the DECT group. In our study, the differences in the CNR and FOM values of liver, spleen, and portal vein between the SECT and DECT groups were not statistically significant. The images obtained using a third-generation dual-source scanner in SECT and DECT modes were comparable in our study. The result was similar to that from the study done by Lenga et al. [23], which showed no significant difference in objective image quality in images obtained using SECT and DECT modes in a third-generation dualsource scanner. In another study done by Lenga et al. [24], in the evaluation of chest CT, there were no statistically significant differences in CNR values of descending thoracic aorta, pulmonary trunk, and thyroid gland as well as the FOM value of the pulmonary trunk between SECT and DECT groups of a third-generation scanner.

Hence, DECT can be considered in all scenarios because it can increase both lesion detection and characterization and has the capacity to decrease artifacts and possibly contrast media dose, all while maintaining or even decreasing the radiation dose to the patient. The spectral imaging potential of DECT can be utilised routinely in abdominal DSCT.

Our study had some limitations. This study was done on the dual-source dual-energy technique. Hence, the effective radiation dose values obtained cannot be generalised to other techniques of dual-energy CT like rapid 
kilovolt switching, multilayer detector technique, etc., which might show different results. The study was a single institutional study with limited subjects. Further largescale studies and meta-analysis are needed to confirm our results.

\section{Conclusions}

In third-generation dual-source dual-energy CT there is a significant reduction in the effective radiation dose to the patient compared to single-energy CT in matched cohorts. In our study, the routine abdominal examination protocols in third-generation DSCT using dual-energy mode resulted in an approximately 58\% dose reduction compared to single-energy CT with similar image quality. Therefore, the quantitative imaging potential of DECT can be routinely utilised with decreased radiation dose in third-generation dual-source dual-energy CT in abdominal imaging.

\section{Conflict of interest}

The authors report no conflict of interest.

\section{References}

1. Sodickson AD, Keraliya A, Czakowski B, et al. Dual energy CT in clinical routine: how it works and how it adds value. Emerg Radiol 2021; 28: 103-117.

2. Uhrig M, Simons D, Kachelrieß M, et al. Advanced abdominal imaging with dual energy $\mathrm{CT}$ is feasible without increasing radiation dose. Cancer Imaging 2016; 16: 15.

3. Wichmann JL, Hardie AD, Schoepf UJ, et al. Single- and dual-energy $\mathrm{CT}$ of the abdomen: comparison of radiation dose and image quality of 2nd and 3rd generation dual-source CT. Eur Radiol 2017; 27: 642-650.

4. De Cecco CN, Darnell A, Macias N. Second-generation dual-energy computed tomography of the abdomen: radiation dose comparison with 64- and 128-row single-energy acquisition. J Comput Assist Tomogr 2013; 37: 543-546.

5. The 2007 recommendations of the International Commission on Radiological Protection. ICRP publication 103. Ann ICRP 2007; 37: 1-332.

6. Christner JA, Kofler JM, McCollough CH. Estimating effective dose for CT using dose-length product compared with using organ doses: consequences of adopting International Commission on Radiological Protection publication 103 or dual-energy scanning. Am J Roentgenol 2010; 194: 881-889.

7. Li Y, Li Y, Jackson A, et al. Comparison of virtual unenhanced CT images of the abdomen under different iodine flow rates. Abdom Radiol 2017; 42: 312-321.

8. De Ceccoa CN, Muscogiuria G, Schoepf UJ, et al. Virtual unenhanced imaging of the liver with third-generationdual-source dual-energy CT and advanced modelled iterativereconstruction. Eur J Radiol 2016; 85: 1257-1264.

9. Schindera ST, Nelson RC, Mukundan S Jr, et al. Hypervascular liver tumors: low tube voltage, high tube current multi-detector row CT for enhanced detection - phantom study. Radiology 2008; 246: 125-132.

10. Chiro GD, Brooks RA, Kessler RM, et al. Tissue signatures with dual-energy computed tomography. Radiology 1979; 131: 521-523.

11. Millner MR, McDavid WD, Waggener RG, et al. Extraction of information from CT scans at different energies. Med Phys 1979; 6: 70-71.

12. Kalender WA, Perman WH, Vetter JR, et al. Evaluation of a prototype dual-energy computed tomographic apparatus. I. Phantom studies. Med Phys 1986; 13: 334-339.

13. Kelcz F, Joseph PM, Hilal SK. Noise considerations in dual energy CT scanning. Med Phys 1979; 6: 418-425.
14. Smith-Bindman R, Lipson J, Marcus R, et al. Radiation dose associated with common computed tomography examinations and the associated lifetime attributable risk of cancer. Arch Intern Med 2009; 169: 2078-2086.

15. Patient safety: radiation dose in X-ray and CTexams. Available at: http://www.radiologyinfo.org/en/safety/?pg=sfty_xray\#part3 (Accessed: 28.04.2015).

16. Primak AN, Giraldo JC, Eusemann CD. Dual-source dual-energy CTwith additional tin filtration: dose and image quality evaluation in phantoms and in vivo. AJR Am J Roentgenol 2010; 195: 1164-1174.

17. Purysko AS, Primak AN, Baker ME. Comparison of radiation dose and image quality from single-energy and dualenergy CT examinations in the same patients screened for hepatocellular carcinoma. Clin Radiol 2014; 69: e538-544.

18. Schmidt D, Söderberg M, Nilsson M, et al. Evaluation of image quality and radiation dose of abdominal dual-energy CT. Acta Radiol 2018; 59: 845-852.

19. Gordic S, Morsbach F, Schmidt B, et al. Ultralow-dose chest computed tomography for pulmonary nodule detection: first performance evaluation of single energy scanning with spectral shaping. Invest Radiol 2014; 49: 465-473.

20. Schmid AI, Uder M, Lell MM. Reaching for better image quality and lower radiation dose in head and neck CT: advanced modeled and sinogram-affirmed iterative reconstruction in combination with tube voltage adaptation. Dentomaxillofac Radiol 2017; 46: 20160131.

21. Chen CY, Hsu JS, Jaw TS, et al. Split-bolus portal venous phase dualenergy CT urography: protocol design, image quality, and dose reduction. AJR Am J Roentgenol 2015; 205: W492-W501.

22. Tawfik AM, Kerl JM, Razek AA, et al. Image quality and radiation dose of dual-energy CT of the head and neck compared with a standard 120-kVp acquisition. Am J Neuroradiol 2011; 32: 1994-1999.

23. Lenga L, Trapp F, Albrecht MH, et al. Single- and dual-energy CT pulmonary angiography using second- and third-generation dual-source CT systems: comparison of radiation dose and image quality. Eur Radiol 2019; 29: 4603-4612.

24. Lenga L, Leithner D, Peterke JL, et al. Comparison of radiation dose and image quality of contrast-enhanced dual-source CT of the chest: single-versus dual-energy and second-versus third-generation technology. Am J Roentgenol 2019; 212: 741-747. 